Symmetry Considerations on the Stiffness and Eigen Deformations of Plant Fibers and Structures Made of Them

\author{
Petri P. Kärenlampi \\ University of J oensuu \\ Faculty of Forestry \\ Box 111 \\ FIN-80101 J oensuu \\ petri.karenlampi@joensuu.fi \\ tel. $+358-50-3711851$ \\ fax $+358-13-2514422$ \\ PACS \\ 87.85.jf Biomaterials \\ 02.20.-a Group theory \\ 62.20.de Elastic moduli
}

\title{
Symmetry Considerations on the Stiffness and Eigen Deformations of Plant Fibers and Structures Made of Them
}

\begin{abstract}
Plant fibers with nonzero microfibril angle show no plane reflection symmetries, the groups of spatial symmetry transformations consisting of rotations only. The spatial point symmetry group of any material made of such fibers is of order which is half of the order of the symmetry group of the corresponding orthotropic material. However, materials consisting of fibers show similar degeneracies of stiffness Eigenvalues as the non-fibrous materials. Stiffness degeneracies appear to be controlled by the integer exponents of dicycle conditions applied on products of vectors generating symmetry groups. It is found that flat orthotropic sheets always retain their planar shape in Eigen Deformations, whereas those made of fibers with microfibrils do not. Features of the out-of-plane deformations are clarified. Orthotropic material elements experience normal on-axis Eigen Strains only, whereas fibrous bodies with orthotropic fiber alignment may experience on-axis shear strains as Eigen Strains.
\end{abstract}

Keywords:

Group theory, representation theory, material symmetries, irreducible representations, dimensionality. 


\section{Introduction}

Plant fibers are widely used in construction, in packages, in information carrier products, and in a variety of engineering applications. Plant fiber products often provide a high stiffness in relation to mass, as well as desirable thermal, optical and acoustic properties.

Plant fibers, consisting of helically wound microfibrils, have a rather special structure. Thus the symmetries of a plant fiber, as well as those of a plant fiber product, are somewhat peculiar. Spatial symmetry transformations may be translations, reflections, rotations or inversions. There are conservation laws related to symmetries [1-4]. In other words, properties are retained in symmetry transformations. Further, quite a few properties of structures may be deduced on the basis of the symmetries of the system, using group theory and group representation theory [1-6].

In this paper, we will investigate the spatial symmetries of plant fibers, as well as a few implications of their symmetries. In particular, we will focus in degeneracies of stiffness Eigenvalues of plant fiber materials on the one hand, and on Eigen Deformations of such materials on the other hand.

First, we will briefly review group theory, and discuss spatial symmetry transformations of orthotropic, transversely square-symmetric, and transversely isotropic materials. Then, we will consider a plant fiber composed of helically wound microfibrils, and the symmetry transformations of such a fiber. We find a symmetry group which differs from that of the transversely square-symmetric material, plane reflection symmetries being absent.

Symmetries of structures composed of plant fibers will be discussed. Properties of such structures can be viewed as effects induced by the properties of the plant fibers on the one hand, and the arrangement of the fibers within the body on the other hand. According to the classical symmetry principle, or Curie's principle, an effect always is at least as symmetric as the cause $[7,8]$. Structures made of plant fibers may or may not show more symmetries than the fibers themselves.

Degeneracies in stiffness Eigenvalues will be discussed as a function of the properties of any symmetry group of a continuum material composed of plant fibers. Finally, the structure of a body, together with the potential field within the body, is discussed as a cause, and Eigen Deformation as the effect. All symmetries of the cause are retained in deformed bodies, which however may display additional symmetries.

\section{Group Theory and Representation Theory}

A group is a set of elements showing a few special properties. In particular, any group member acting on another group member produces a group member. This property is called Closure. The group operation is associative, i.e. $(X Y) X=X(Y Z)$. Every group has an identity element, $I Y=Y I=Y$, and every element has an inverse element, $Y^{-1} Y=Y Y^{-1}=I$. The order of a group is the number of group elements. The order of an element is the power of the element producing Identity. The order of the group, as well as the orders of the elements often need to be known in order to identify the group. Group elements may be divided into conjugacy classes, members of any class being connected by similarity transformations $X=Y^{-1} Z Y$. The conjugacy classes do not overlap $[3,5,6]$. 
Group elements are often represented by matrices. A representation is reducible if the vector space where it acts on contains a proper subspace, closed under the action of the matrix. Otherwise the representation is Irreducible. A number of theorems apply to Irreducible Representations (Irreps); among others, orthogonality, completeness, and dimensionality theorems $[3,5,6]$. Using such theorems, a character table can be constructed for any discrete group. The character refers to the trace of a matrix, which is retained in unitary similarity transformations. Thus all the members within a conjugacy class have the same character within any Irrep. The number of Irreps equals the number of classes, and thus the character table is a square matrix.

Any representation of a particular group can be composed of Irreps of the group. This can often be done on the basis of the character table. For a particular representation, acting on a vector space spanned by some basis vectors, the character of any conjugacy class must be clarified. This character must be the sum of the characters of the Irreps for that particular conjugacy class, and the same Irreps constituting the representation must satisfy this requirement simultaneously for all conjugacy classes.

Regarding the compatibility of properties, the dimensionality of the Irreps is of outmost importance. Compatible properties "mix" during symmetry transformations. In other words, they transform with multidimensional Irreps. In the particular case of normal modes, vibrations transforming with the same Irrep are degenerate, and the dimensionality of the Irrep indicates the degree of degeneracy. Abelian groups (all elements commuting) have only one-dimensional Irreps $[3,5,6]$.

\section{Symmetry Groups of Materials and Structures}

\subsection{Orthotropy and Transverse Square-Symmetry}

A two-dimensional orthotropic material retains its properties in four symmetry operations: two line reflections, one with respect to each of the material symmetry axis, a rotation by $\pi$, and Identity. Thus the group multiplication table consists of 16 elements. There are two non-isomorphic groups of order 4 . This one is the so called "Vierergruppe", or $4 / 2$. The group multiplication table is symmetric. Thus the group is Abelian, and has only one-dimensional Irreps.

A three-dimensional orthotropic material retains its properties in eight symmetry operations: three plane reflections, one with respect to each of the material symmetry planes, three rotations by $\pi$, Identity, and parity inversion (or point reflection). Thus the group multiplication table consists of 64 elements. Seven of the symmetry operations are of order 2 . This identifies the group as $8 / 3$, according to nomenclature of Thomas and Wood [9], or prismatic group $m m m=D_{2 h}=V_{h}$. The group is Abelian, and thus has only one-dimensional Irreps.

In comparison to the three-dimensional orthotropic material, a transversely squaresymmetric material has eight more symmetry operations. It is invariant in rotations by $\pm 2 \pi / 4$, as well as reflections with respect to planes diagonal to the principal material axis. In addition, the symmetry group includes these transformations combined with reflection with respect to the plane of square-symmetry. Four of the symmetry operations are of order four, 11 are of order two. This identifies the group as $16 / 6$, also know as prismatic group $4 / \mathrm{mmm}=\mathrm{D}_{4 \mathrm{~h}}[3,10]$. 
The group elements of $16 / 6$ form ten conjugacy classes, and thus the group has ten Irreps. Character table for the group is shown in Table 1. In Table 1, we denote rotations by $2 \pi / n$ with respect to axis $x_{i}$ as $n_{x i}$, and plane reflections with respect to an $\{i, j\}$ - co-ordinate plane as $M_{x i j}$. P rime refers to reversal of angular sign. Plane reflection with respect to a plane along $x_{3}$-axis and diagonal to axis $\{i, j\}$ is denoted as $m_{x i 3}$. $i$ refers to parity inversion. Double bar indicates reflection with respect to the plane of square-symmetry, here the $\{1,2\}$ - plane, i.e. $\overline{\overline{4_{x 3}}}=M_{x 12} 4_{x 3}$. Roman capitals $\mathrm{A}$ and $\mathrm{B}$ refer to one-dimensional Irreps, and $\mathrm{E}$ to a twodimensional Irreps.

\subsection{Cube-Symmetry}

Trying to construct the Group Multiplication Table for cube-symmetry, one readily finds that this is a large group. The symmetry group of a cube is of order 48. Thus the Group Multiplication Table contains 2304 elements. The symmetry group is denoted Cubic or Octahedral Group $m 3 m=O_{h}[3,10]$. Eight of the group members are of order six, 12 of order four, eight of order three and 19 of order two.

The group members form ten conjugacy classes, and thus the group has ten Irreps. Character table for the group is shown in Table 2. Table 2 is kept brief and simple by noting a class of six rotations by $2 \pi / 2$ as $6{ }^{*} C_{2}$. These take place with respect to a line from the center of a cube edge to the center of the opposite edge. Another brief notation is ${ }^{*} C_{3}$. This refers to eight rotations by $2 \pi / 3$, with respect to cube diagonals. The last column of the Table refers to the latter, followed by parity inversion, and the second last to rotations by $\pm 2 \pi / 4$, followed by parity inversion.

\subsection{Two-dimensional and Transverse Isotropy}

Isotropy actually corresponds to a continuous symmetry group of infinite order. However, we are able to describe in-plane isotropy in terms of a finite symmetry group, considering that a few simple laminate structures display quasi-isotropic behavior. The simplest of these is the so called -60/0/60 - laminate, where layers are aligned with angular intervals of $2 \pi / 3$. In two dimensions, this kind of a structure has 12 symmetry operations. Two of them are of order six, two of order three, and seven of order two. This identifies the group as 12/3, according to nomenclature of Thomas and Wood [9], also know as Dihedral Group 622 $=D_{6}$. It appears to be isomorphic to group $6 \mathrm{~mm}=\mathrm{C}_{6 \mathrm{v}}[9,3,10]$.

The group members form six conjugacy classes, and thus the group has six Irreps. Character table for the group 12/3 is shown in Table 3 . In Table 3, we denote a plane reflection with respect to a plane aligned within an axis of rotation symmetry with $\mathrm{M}_{\mathrm{i}}$, and a reflection with respect to a plane bisecting the angles between the previous with $m_{i}$.

In addition to the symmetry operations of the corresponding two-dimensional case, a three-dimensional orthotropic body with transverse isotropy has symmetry operations combining the previous with reflection with respect to the plane of isotropy. This applies to all the elements of group 12/3, the outcomes happening to include rotations with respect to axis within the plane of isotropy, as well as parity inversion. Thus the increment of dimensionality exactly doubles the order of the symmetry group. There are six elements of order six, two elements of order three, and 15 elements of order two. On the basis of this, the symmetry group can be identified as $24 / 4$, which appears to be isomorphic to group $6 / \mathrm{mmm}=D_{6 h}[9,3,10]$.

The group members form 12 conjugacy classes, and thus the group has 12 Irreps. Character table for the group 24/4 is shown in Table 4. In Table 4, notations are the same as in Tables 2 and 3 , double bar indicating reflection with respect to the plane of isotropy. 


\subsection{A Fiber with Helically Wound Microfibrils}

Let us then discuss a plant fiber, consisting of helically wound microfibrils. To provide an example, a microphotograph of cellular tissue of softwood is shown in Fig. 1. A polarized-light microphotograph of an individual fiber with helical striations, chemically released from the structure, is shown in Fig. 2 . If the microfibril angle were neglected, an intact fiber might show transverse square-symmetry. However, any of those plane reflections which are symmetry operations for square-symmetry will reverse the microfibril angle. On the other hand, any line reflection can be written in terms of two consequent plane reflections. Thus line reflections retain the microfibril angle. Similarly, any rotation can be written in terms or two plane reflections [11, 12, 13]. Thus rotations preserve the microfibril angle. Any point reflection (or parity inversion) can be written in terms of three plane reflections. Thus point reflections do reverse the microfibril angle. Those symmetry transformations for the transversely isotropic material which correspond to odd numbers of plane reflections do not constitute symmetry transformations for the plant fiber with nonzero microfibril angle.

Eight of the symmetry transformations of the transversely square-symmetric material remain for the plant fiber. Two of them are of order four, and five are of order two. This identifies the symmetry group as $8 / 4$, also known as Dihedral Group

$422=D_{4}$. It appears to be isomorphic to groups $4 m m=C_{4 v}$ and $\overline{4} 2 m=D_{2 d}=V_{d}[9,3$, 10]. The group elements form five conjugacy classes, and thus the group has five Irreps. A character table for the group 8/4 is shown in Table 5.

\subsection{A Body with All-Parallel Fibers}

In the absence of the effect of microfibril angle, a body with all-parallel fibers might show transverse square-symmetry. Microfibril angle however being reversed in plane reflections, the symmetry group of a structure consisting of all-parallel fibers is the same as the symmetry group of a single fiber, i.e. $8 / 4$.

\subsection{Fibrous Structures with Orthotropy in Two Dimensions}

A structure with all fibers parallel to a plane and with orthotropic orientation distribution of fibers within the plane, this distribution invariant with respect to the co-ordinate perpendicular to the plane, would show the symmetries of threedimensional orthotropy, i.e. symmetry group $8 / 3$. But, any plane reflection, as well as any point reflection, reverses the microfibril angle. Then, the symmetry operations consist of three rotations by $\pi$, one with respect to the center line of each of the two orthotropic symmetry planes, and the third with respect to an axis perpendicular to them, and Identity. Three of the four symmetry operations are of order two. The symmetry group is $4 / 2$, or the Vierergruppe, and it has only onedimensional Irreps.

A structure with all fibers parallel to a plane, with orthotropic orientation distribution of fibers within the plane, but this distribution not invariant with respect to the coordinate perpendicular to the plane, is not invariant in rotations with respect to the center line of each of the two orthotropic symmetry planes. Thus the symmetry group is of order two. There is only one group of this order, and it is Abelian.

A structure with all fibers parallel to a plane and with square-symmetric orientation distribution of fibers within the plane, this distribution invariant with respect to the co-ordinate perpendicular to the plane, would show transverse square-symmetry, in addition to those of three-dimensional orthotropy. The symmetry group would be $16 / 6$. However, any plane reflection, as well as any point reflection, reverses the microfibril angle. Eight of the 16 symmetry operations of group 16/6 retain the microfibril angle, the symmetry group thus being the same as that of a single fiber, i.e. $8 / 4$. 
A structure with all fibers parallel to a plane, with square-symmetric orientation distribution of fibers within the plane, but this distribution not invariant with respect to the co-ordinate perpendicular to the plane, is not invariant in rotations with respect to the center line of each of the two orthotropic symmetry planes. Neither is it invariant in two consequent plane reflections, one of the reflection planes being that of fiber alignment. The symmetry group is of order four. Two of the symmetry operations are of order four, and one of order two. This identifies the group as 4/1, or cyclic group $\mathrm{C}_{4}$. The group is Abelian, and thus has only one-dimensional Irreps.

For a structure with all fibers parallel to a plane and with isotropic orientation distribution of fibers within the plane, any rotation within the plane would be a symmetry operation. Also any plane reflection within the sheet plane would retain the random distribution of fiber orientation. Such a transverse isotropy can be characterized by a discrete symmetry group which includes rotations corresponding to those which are symmetry operations of quasi-isotropic laminates. The simplest of these is the $-60 / 0 / 60$ - laminate, where the symmetry group can be identified as $24 / 4$, which appears to be isomorphic to group $6 / \mathrm{mmm}=\mathrm{D}_{6 \mathrm{~h}}[9,3,10]$.

However, any plane reflection would reverse the microfibril angle. The same applies to any point reflection, as well as any operation which contains an odd number of plane reflections. The resulting discrete symmetry group is of order 12 . Two of the group elements are of order six, two of order three, and seven of order two. This identifies the group as 12/3, according to nomenclature of Thomas and Wood [9], also know as Dihedral Group 622D ${ }_{6}$. It appears to be isomorphic to group $6 \mathrm{~mm}=\mathrm{C}_{6 \mathrm{v}}[9,3,10]$.

The group elements of 12/3 form six conjugacy classes, and thus the group has six Irreps. Character table for the group is shown in Table 6 , where just the column headings differ from those of Table 3 . In Table 6 , we denote line reflection (or rotation) with respect to an axis within the $x_{1}-x_{2}$-plane with of angular alignment $2 \pi / \mathrm{i}$ from co-ordinate axis $x_{2}$ with $L_{1}$.

Within a structure with all fibers parallel to a plane and with isotropic orientation distribution of fibers within the plane, the fiber alignment is not necessarily orthotropic in three dimensions. Even if the fiber alignment is isotropic within a plane, it does not need to be invariant as a function of the co-ordinate perpendicular to the plane, since the distribution of axial fiber rotations may vary. In case it does not vary symmetrically with respect to the central plane of the sheet, out-of plane rotations are not symmetry operations for the sheet, and the symmetry group corresponding to the 60/0/60 - laminate is of order 6 . Two of the elements are of order six, two of order three, and one of order two. This Identifies the symmetry group as 6/1, which is Abelian.

\subsection{Fibrous Structures with Orthotropy in Three Dimensions}

As mentioned above, three-dimensional orthotropy corresponds to symmetry group $8 / 3$. However, a body consisting of fibers with helically wound microfibrils, the fibers aligned according to three-dimensional orthotropy, corresponds to symmetries of the Vierergruppe 4/2, which is Abelian.

As well it was mentioned above that transverse square-symmetry, in addition to three-dimensional orthotropy, corresponds to symmetry group 16/6. A body consisting of fibers with helically wound microfibrils, the fibers aligned according to three-dimensional orthotropy, corresponds to symmetry group $8 / 4$, which is the same as the symmetry group of a single fiber.

Cube-symmetry corresponds to symmetry group $O_{h}$, which is of order 48 . The order of the symmetry group for fibers aligned according to cube-symmetry is 24 , half of the symmetry operations shown in Table 2 not being applicable. Five conjugacy classes remain; the character table can easily be found from the left part of Table 2 . 


\section{Degeneracies in Stiffness Eigenvalues}

W ithin a continuum material, deformations can be described in terms of strains: partial derivatives of displacements with respect to co-ordinates. In a threedimensional body, the strain tensor has nine components. All deformations within a body can be described in terms of the strain components. Thus the number of the strain components corresponds to the number of normal modes of vibration.

W ithin a co-ordinate system, stresses can be given as partial derivatives of forces with respect to areas perpendicular to co-ordinate axis. The number of stress tensor components is the same as the number of strain tensor components. Strains are related to stresses through Stiffnesses. The Stiffness Matrix thus is a 9*9 matrix in the case of a three-dimensional body. The Stiffness Matrix may be diagonalized in terms of a Unitary Transformation. Such a diagonalization results as nine (or four in two dimensions) stiffness Eigenvalues, as well as strain and stress components expressed in a co-ordinate system called Normal Co-ordinates.

In normal co-ordinates, one can easily raise an argument regarding conservation of energy. In the particular case of an elastic body, the time derivative of the sum of kinetic energy and strain energy must be zero. The energy components can be written in terms of body dimensions, stiffnesses, strains, and time derivatives of strains. This applies separately for any co-ordinate direction in the normal coordinates, and leads to an Equation of strain and second time derivative of strain

$\ddot{\varepsilon}=-\omega_{i}{ }^{2} \varepsilon$

the solution of which is a cyclical function with angular frequency $\omega_{\mathrm{i}}$,

$$
\omega_{\mathrm{i}}=\frac{\mathrm{S}_{\mathrm{i}}}{\mathrm{D}} \sqrt{\frac{\mathrm{Q}_{\mathrm{i}}}{\rho}}
$$

where $s_{i}$ is a direction-dependent geometry factor, $D$ is linear size, $Q_{i}$ is stiffness Eigenvalue, and $\rho$ is mass density of the material. Repeated index does not imply summing in Eq. (2).

The nine Eigenfrequencies are not necessarily all distinct. In such a case the normal modes of vibration are said to be degenerate. We find from Eq. (2) that any Eigenfrequency depends on the geometry and size of the Representative Material Element under discussion. The stiffness Eigenvalue $Q_{i}$ does not depend on size or geometry. It solely characterizes the properties of the material.

\section{Representations for Non-Fibrous Materials}

In order to utilize character tables above for the composition of a representation of the group on a nine-dimensional strain vector space, we should determine the character for any conjugacy class of symmetry operations acting on such a space. Firstly, the character of the Identity is of course the same as the number of basis vectors spanning the vector space. The same applies to inversion, which retains all strain components. In the case of a nine-dimensional strain space, the trace of any rotation matrix is $4 \cos ^{2} \theta+4 \cos \theta+1$. It is further worth noting that three-dimensional orthotropy corresponding to symmetry group $8 / 3$, which is Abelian, and thus contains only one-dimensional Irreps, does not induce any stiffness degeneracies. In other words, no stiffness degeneracies are due to orthotropic material symmetry. Symmetry of the strain and stress tensors makes that there are six distinct stiffness Eigenvalues. 


\subsection{Transverse Square-Symmetry}

Regarding transverse square-symmetry, in addition to three-dimensional orthotropy, a consequence of the character of the inversion being the same as the dimensionality of the strain space, the representation of the symmetry group 16/6 on nine-dimensional strain space must consist of g-Irreps, and not include any uIrreps of Table 1 . Since the trace of any rotation matrix is $4 \cos ^{2} \theta+4 \cos \theta+1$, the character of both $2_{x i}$ and $4_{x i}$ is unity. In order to the character $2_{x 3}$ become unity, simultaneously as the character of the Identity is nine, Irrep $E_{g}$ (Table 1) must appear two times in the representation, and the combined appearance of the rest of the $g$-Irreps must be five.

$\mathrm{m}_{\mathrm{x} 13}$ exchanges co-ordinates 1 and 2 . The only strain component it retains is $\varepsilon_{33}$, and thus the character is 1 . The only representation which gives the correct character for all these conjugacy classes is $D=2 A_{1 g} \oplus A_{2 g} \oplus B_{1 g} \oplus B_{2 g} \oplus 2 E_{g}$. It is straightforward to verify that the characters of the rest of the group members do comply with this representation, these characters all being equal to unity.

Thus we find that transverse square-symmetry induces two two-fold degeneracies in stiffness Eigenvalues. This is somewhat surprising since it has been recently shown that square-symmetry does not induce any degeneracies of stiffness Eigenvalues in the case of a four-dimensional strain space [14]. A first guess might be that the degenerate Eigenvalues might be among those which do not exist in the case of a two-dimensional material. It can actually be verified that $Q_{1313}=Q_{2323}$, and $Q_{3131}=Q_{3232}$ [14]. Further considering the symmetry of the stress tensor and the strain tensor, which are not due to material symmetry, we find that there are five distinct stiffness Eigenvalues.

\subsection{Cube-Symmetry}

Again, the character of the Identity, as well as the character of the Inversion equals the number of basis vectors in spanning the nine-dimensional vector space, i.e. nine. Thus we find that the representation of the group on the strain vector space must consist of $\mathrm{g}$-Irreps of Table 2 only, and not include any u-Irreps. One of the members of the class $C_{2}$ can be given as a combination of two reflections, namely $M_{x 12} m_{x 23}$. The character of this transformation is calculable by transforming coordinates, as well as displacements, and composing transformed strain components of them. The transformation does preserve one of the strain components. No one changes sign, and thus the character is one. One of the members of class $C_{3}$ can be given as $m_{x 23} m_{\times 32}$. The character of this transformation can similarly be clarified as zero. The latter indicates that the number of A-Irreps in the Representation must equal the number of E-Irreps. This number cannot be zero. If it would, the Representation would consist of T-Irreps only, and the character of $2_{x i}$ could not equal unity. The only number which gives simultaneously unity for $2_{x i}$ and nine for Identity is one.

Then, two questions remain. Which one of the Irreps $A_{1 g}$ and $A_{2 g}$ appears in the Representation? The combined number of $T_{1 g}$ and $T_{2 g}$ must be two, but how may of each? 
Now, there are two ways of making the character of $4_{x i}$. Either $A_{1 g} \oplus T_{1 g} \oplus T_{2 g}$ or $A_{2 g} \oplus 2 T_{1 g} \quad$ must appear in the Representation. On the other hand, $m_{x 13}$ exchanges co-ordinates 1 and 2 . The only strain component it retains is $\varepsilon_{33}$, and the character is 1. Thus either $A_{1 g} \oplus T_{1 g} \oplus T_{2 g}$ or $A_{2 g} \oplus 2 T_{2 g}$ must appear in the Representation. Only the former works for $4_{x i}$. Thus the Representation is $D=A_{1 g} \oplus E_{g} \oplus T_{1 g} \oplus T_{2 g}$.

We find that in the case of cube-symmetry, there is one two-fold degeneracy, and two three-fold degeneracies in stiffness Eigenvalues due to material symmetry. The three-fold degeneracies obviously refer to shear stiffnesses. Considering also the symmetry of the stress and strain matrices, all shear stiffnesses must be equal. The two-fold degeneracy corresponds to stiffness Eigenvalues related to normal strains and normal stresses. Thus there are three distinct stiffness Eigenvalues.

\subsection{Transverse Isotropy}

Regarding the transverse quasi-isotropy group 24/4, the character of the Identity, as well as the character of the inversion, equals the number of basis vectors spanning the nine-dimensional vector space, i.e. nine. Thus we find from Table 4 that the representation of the group on the strain vector space must consist of g-Irreps only, and not include any u-Irreps. Again, the trace of any rotation matrix is $4 \cos ^{2} \theta+4 \cos \theta+1$. Thus the character of $2_{x i}$ is unity, $3_{x i}$ is zero, and $6_{x i}$ four. The Representation must include two-dimensional Irreps, as many as one-dimensional, in order to make the character of $3_{x i}$ zero. The character of the Identity then requires that there are three two-dimensional and three one-dimensional Irreps. The characters of $2_{x i}$ and $\sigma_{x i}$ become right if there are three A-Irreps, two $E_{1 g}$ and one $E_{2 g}$. In order to separate between the A-Irreps, we have to study the reflections. $M_{1}$ and $m_{2}$ are with respect to co-ordinate axis. Thus they change the sign of $\varepsilon_{12}$ and $\varepsilon_{21}$ but retain $\varepsilon_{11}, \varepsilon_{22}$ and $\varepsilon_{33}$. In particular, $M_{1}$ also changes the sign of $\varepsilon_{13}$ and $\varepsilon_{31}$, but retains $\varepsilon_{23}$ and $\varepsilon_{32}$. Thus the character of this transformation is unity. The only combination of Irreps compatible with these characters is $D=2 A_{1 g} \oplus A_{2 g} \oplus 2 E_{1 g} \oplus E_{2 g}$.

We find that transverse isotropy induces three two-fold degeneracies in stiffness Eigenvalues. Thus one additional degeneracy appears, in relation to the transverse square-symmetry: one of the Eigenvalues corresponding to normal strains equals the shear stiffness within the plane of Isotropy. There are four distinct stiffness Eigenvalues.

\subsection{Three-Dimensional Isotropy}

Isotropy actually corresponds to a continuous Symmetry Group of infinite order. It obviously would be possible to generalize the two-dimensional laminate treatments into three dimensions. This would, create discrete symmetry groups of rather large order, with huge group multiplication tables. One of the candidates for a discrete group which might be used to describe three-dimensional isotropy may be the Icosahedral group of order 120. 
Fortunately, isotropy is a rather trivial case, regarding degeneracies of stiffness Eigenvalues. There are two distinct stiffness Eigenvalues. How is this supposed to be reflected in the dimensionality of the Irreps constituting the Natural Representation of the Symmetry Group, acting on the nine-dimensional strain space? Recalling that the stress tensor and the strain tensor are symmetric by definition, and not due to material symmetry, the six Shear Stiffnesses transform according to two different Irreps. Then, two of the stiffness Eigenvalues corresponding to normal strains are degenerate, and similar to the shear stiffnesses. Thus the representation should contain one five-dimensional Irrep and one three-dimensional /rrep. Dimensionality of the strain space then requires a third Irrep, which must be one-dimensional.

\section{Representations for Fibrous Materials}

\subsection{A Body with All-Parallel Fibers}

The trace of any rotation matrix being $4 \cos ^{2} \theta+4 \cos \theta+1$, the character of both $2 x i$ and $4_{x i}$ is unity. Regarding $2_{x 3}$, this requires the two-dimensional Irrep $E$ to appear two times in the Representation (Table 5 ). Rest of the rotation characters require the Irrep $A_{1}$ to appear two times in the Representation. The only combination of Irreps compatible with these characters $D=2 A_{1} \oplus A_{2} \oplus B_{1} \oplus B_{2} \oplus 2 E$. It appears that the Stiffness Degeneracies are the same as those of transverse square-symmetry (group 16/6), even if the symmetry group is $8 / 4$.

\subsection{Fibrous Structures with Orthotropy in Two Dimensions}

A structure with all fibers parallel to a plane and with square-symmetric orientation distribution of fibers within the plane corresponds to symmetry group $8 / 4$, and has the character table shown in Table 5. The Representation of this group in the ninedimensional strain space thus is the same as in the case of a body of all-parallel fibers, and even the Stiffness Degeneracies are the same, being equal to those corresponding to transverse square-symmetry.

For a structure with all fibers parallel to a plane and with isotropic orientation distribution of fibers within the plane, the symmetry can be described in terms of group $12 / 3$, and the character table is shown in Table 6 . The character of 3 xi being zero, we find that the number of two-dimensional Irreps must equal the number of one-dimensional Irreps. The character of the Identity requires that this number must be three. The characters of $2_{x 1}$ and $2_{x 2}$ being unity require that the one-dimensional irreps consist of two times $A_{1}$ and once $A_{2}$. Then, the character of $2_{x 1}$ being unity requires that the two-dimensional irreps are two times $E_{1}$ and once $E_{2}$. Thus the Representation is $D=2 A_{1} \oplus A_{2} \oplus 2 E_{1} \oplus E_{2}$. Thus the stiffness degeneracies are the same as those of transverse isotropy, even if the symmetry group is $12 / 3$ instead of $24 / 4$.

\subsection{Fibrous Structures with Orthotropy in Three Dimensions}

Transverse square-symmetry, in addition to three-dimensional orthotropy, corresponds to symmetry group 16/6. A body consisting of fibers with helically wound microfibrils, the fibers aligned according transverse square-symmetry, in addition to three-dimensional orthotropy, corresponds to symmetry group 8/4, which is the same as the symmetry group of a single fiber. A body with all-parallel fibers actually is one special case of this kind of a structure. Another special case is a body where all fibers are parallel to a plane and with an invariant square-symmetric orientation distribution within the plane. All these structures show two two-fold stiffness degeneracies, and have five distinct stiffness Eigenvalues. 
Transverse quasi-isotropy, in addition to three-dimensional orthotropy, corresponds to symmetry group 24/4. A body consisting of fibers with helically wound microfibrils, the fibers aligned according transverse square-symmetry, in addition to three-dimensional orthotropy, corresponds to symmetry group 12/3. A body where all fibers are parallel to a plane and with an isotropic orientation distribution within the plane is a special case of this kind of a structure. All these structures show three two-fold stiffness degeneracies, and four distinct stiffness Eigenvalues.

Cube-symmetry corresponds to a symmetry group of order 48 . A body consisting of fibers with helically wound microfibrils, the fibers aligned according cube-symmetry, corresponds to a symmetry group of order 24 . A Representation of this group on a nine-dimensional strain vector space can be composed on the basis of the Character Table 2 . The character of 3 xi being zero, we find that the number of twodimensional Irreps must equal the number of one-dimensional Irreps. The character of $2_{x i}$ being unity, and the character of Identity being nine, that number must be one, and in addition, there must be two three-dimensional Irreps. The character of $4_{x i}$ being unity, either $A_{1} \oplus T_{1} \oplus T_{2}$ or $A_{2} \oplus 2 T_{1}$ must appear in the Representation. Thus the possible Representations are $D=A_{1} \oplus E \oplus T_{1} \oplus T_{2}$ and $D=A_{2} \oplus E \oplus 2 T_{1}$.

Thus a body consisting of fibers with cube-symmetric alignment has one two-fold degeneracy, and two three-fold degeneracies in stiffness Eigenvalues. Thus the stiffness degeneracies are the same as those of cube-symmetry, even if the symmetry group is of order 24 instead of 48: there are three distinct stiffness Eigenvalues.

Three-dimensional Isotropy might be characterized by the Icosahedral group of order 120 . In the case of material consisting of fibers with helically arranged microfibrils, plane reflection symmetries are nonexistent, and such fibers being aligned according to three-dimensional isotropy, a corresponding finite symmetry group might be the Icosahedral rotation group of order 60 . The results thus indicate that plane reflection and inversion symmetries being absent, the order of any symmetry group becomes reduced by a half. This is likely to be related to the fact that any rotation or line reflection can be produced in terms of two consequent plane reflections $[11,12,13]$.

The above results indicate that the degeneracies in stiffness Eigenvalues might always be the same for a body consisting of fibers with microfibrils as they are for a non-fibrous body with symmetries corresponding to those of symmetries in fiber alignment.

\section{Fibers without Transverse Square-Symmetry}

A plant fiber does not necessarily show transverse square-symmetry, even if the microfibril angle would be neglected. For example, in the case of wood fibers, the tangential and radial cell walls may differ in thickness, as well as in chemical composition [15]. Further, the microfibril angle may not be the same in cell walls with different orientation; it is not uncommon that the microfibril angle is greater in radial cell walls, in comparison to tangential cell walls $[16,17,18]$. In such a case, the symmetry operations of a fiber are limited to three rotations by $\pi$ and Identity. The symmetry group corresponds to the Vierergruppe 4/2. Neglecting the microfibril angle would increase the symmetries into those of group 8/3. Both of these groups are Abelian. A continuous body made of all-parallel fibers has the same symmetries as the single fiber. 
In the case of fibers without transverse square-symmetry, orthotropy in fiber alignment distribution must include an angle of rotation with respect to fiber axis. The symmetry group of three-dimensional orthotropy again is $8 / 3$, but plane reflections and inversion being excluded as symmetry operations, the symmetry group is reduced to the Vierergruppe 4/2.

In the case of transverse square-symmetry in fiber alignment, in addition to orthotropy, the symmetry group would be $16 / 6$, but plane reflections and inversion being excluded as symmetry operations, the symmetry group is reduced to $8 / 4$. Transverse Isotropy would correspond to symmetry group 24/4, which however becomes reduced to $12 / 3$. The symmetry group of cube-symmetry becomes reduced from order 48 to order 24 .

It appears that a structure consisting of all-parallel fibers has different properties depending on whether or not the fibers are transversely square-symmetric: in the symmetric case, there are two two-fold stiffness degeneracies, whereas in the nonsymmetric case there are none. Otherwise, a particular symmetry in fiber alignment always appears to correspond to the same symmetry group, regardless whether the fiber is square-symmetric or not. It is however worth noting that axial rotation of any fiber more easily breaks any fiber alignment symmetry if the fiber is not squaresymmetric.

\section{Eigen Deformations}

We are here denoting Eigen Deformations such changes in size and shape which are results of potential fields homogeneous (and isotropic) in space. Spatial partial derivatives of Eigen Deformations are denoted as Eigen Strains. Examples of such are hygroexpansive and thermal strains. Forces and correspondingly stresses are related to potential gradients. Mechanical strains are related to stresses through constitutive laws, specific to the material. The total deformation of a body depends on internal strains related to internal stresses, strains related to external forces or body forces, as well as Eigen Strains.

According to the classical symmetry principle, or Curie's principle, an effect always is at least as symmetric as the cause $[7,8]$. In the particular case of Eigen Deformations as the effect, the structure of the body, together with the potential field, may be seen as the cause. The potential field inducing Eigen Deformations however being homogeneous, and a potential field always being isotropic, it has all possible symmetries in space. Thus the deformed structure of a body shows at least the symmetries of the undeformed structure of the body, and the symmetries of Eigen Strains are at least those of the material.

The symmetries of a body or structure depend on material symmetries, as well as geometrical symmetries. We will here discuss two kinds of structural geometries: volume elements with geometrical plane reflection symmetries on the one hand, and thin, initially flat sheets on the other hand. A volume element is here understood to be small enough to have essentially homogeneous strain state, whereas the thin sheet is taken as large enough that the strain state may vary within the sheet. Axis or planes of geometrical symmetries may or may not coincide with those of material symmetries. We will first discuss bodies with orthotropic material properties, and then bodies composed of fibers with helically wound microfibrils.

\subsection{Bodies with Geometrical Plane Reflection Symmetries, without Microfibrillar Structure}


A three-dimensional orthotropic material retains its properties in eight symmetry operations, corresponding to group 8/3: three plane reflections, one with respect to each of the material symmetry planes, three rotations by $\pi$, Identity, and parity inversion (or point reflection).

A plane reflection with respect to a co-ordinate plane reverses the sign of any shear strain appearing in the perpendicular co-ordinate planes. In case the geometrical symmetry planes of a volume element coincide with those of the material, the plane reflection symmetries make that on-axis shear strains cannot appear as Eigen Strains for an orthotropic volume element, small enough to have essentially homogeneous strain state. Thus Eigen Deformations of such an element may contain normal deformations only. On the other hand, if no one of the material symmetry axis coincides with any of the geometrical symmetry axis, the body does not have nontrivial spatial symmetry transformations.

It is possible that one but only one of the material symmetry axis coincides with one of the geometrical symmetry axis. In such a case no-one of the material symmetry planes coincides with any of the geometrical symmetry planes, but the system does have a nontrivial spatial symmetry transformation. For a general orthotropic material this operation is a rotation by $\pi$. Consequently, any distortion of the body must repeat itself with an angular distance of $\pi$. Even if on-axis shear strains cannot appear as Eigen Strains for an orthotropic material, the symmetry transformation does allow shear deformations within the co-ordinate system defined by the geometrical symmetry axis of the body.

There are more symmetry operations in some special cases. In case both the material and the body geometry show square-symmetry within the plane perpendicular to the common symmetry axis, rotations by $\pm \pi / 2$ are symmetry operations. Then, any distortion must repeat itself with an angular distance of $\pi / 2$. This does not allow shear deformations within the co-ordinate system defined by the geometrical symmetry axis. In case both the material and the geometry show transverse isotropy, then any rotation with respect to the common symmetry axis is a symmetry operation, and the transverse shape of the body is retained in Eigen Deformations.

Still one more special case is worth considering. In the case of three-dimensional isotropy, any geometrical symmetry axis is a material symmetry axis. Consequently, every geometrical symmetry operation is a symmetry operation for the entire structure. Further, in the case of isotropy, also Eigen Strains are isotropic, and the isotropic body retains its entire geometry in Eigen Deformations.

A flat, thin sheet with orthotropic material properties retains its properties in the symmetry operations corresponding to group $8 / 3$, provided one of the orthotropic material planes coincides with the plane of the sheet, and the sheet has suitable planar geometry. A consequence of the plane reflection symmetries is that the flat sheet must remain flat in Eigen Deformations.

If no one of the orthotropic material planes coincides with the plane of the sheet, any orthotropic plane reflection will change the alignment of the sheet in space. The same applies to any rotation with respect to any symmetry axis of the material, as well as parity inversion. Thus such a structure does not have any nontrivial symmetry transformations in terms of rotations or reflections.

8.2 Volume Elements with Microfibrillar Structure and Geometrical Plane Reflection Symmetries 
A material consisting of fibers with helically wound microfibrils, the fibers aligned according to three-dimensional orthotropy, has symmetries of the Vierergruppe 4/2. The group of symmetry operations contains three mutually perpendicular rotations by $\pi$. Regarding a material element with the same symmetries in its geometry, the symmetries are retained in Eigen Deformations. Thus Eigen Deformations must be periodic within the three rotations by $\pi$. This allows shear deformations, for example.

Additional symmetries in fiber alignment reduce the possible Eigen Deformations. Square-symmetry regarding both the fiber alignment and body geometry makes that rotations by $\pm \pi / 2$ are symmetry operations. This no longer allows shear deformations within the plane of square-symmetry. Isotropy implies that the geometry of the body is retained in Eigen Deformations. In the particular case where one but only one of the material symmetry axis coincides with one of the geometrical symmetry axis, nontrivial symmetry operations consist of rotations with respect to this axis only.

\subsection{Thin Sheets with Orthotropic Orientation of Fibers}

For a structure with all fibers parallel to a plane and with three-dimensional orthotropic orientation distribution of fibers within the plane, the symmetry group is $4 / 2$, or the Vierergruppe. The group contains one in-plane rotation by $\pi$, and two out-of-plane rotations by $\pi$. Any out-of plane rotation converts any concave curvature convex, and vice versa. Thus the out-of-plane rotations as symmetry operations require that if a particular site of the body has concave curvature, the site which corresponds to the image of the first site must have convex curvature, and vice versa. The in-plane angular separation between a site and its image is two times the angle between the site and the axis of out-of-plane rotation. On the other hand, in-plane rotation by $\pi$ is a symmetry operation for this structure. Thus the inplane rotation by $\pi$ must not reverse curvature. The initially flat structure does not need to remain flat in Eigen Deformations, but it may have periodic out-of-plane deformations with an angular period of $\pi$. The curvature must be zero within the axis of the out-of-plane rotation symmetries (or the axis of fiber orientation symmetry). An example of a sheet with Eigen Deformations allowed for group $4 / 2$ is shown in Fig. 3.

Within a structure with all fibers parallel to a plane and with orthotropic orientation distribution of fibers within the plane, the fiber alignment is not necessarily orthotropic in three dimensions. In such a case out-of plane rotations are not symmetry operations for the sheet, and the symmetry group is of order two. In such a case curvature does not need to be reversed as a function of any in-plane rotation. It just needs to be retained in in-plane rotation by $\pi$. This allows the structure to adopt a paraboloidal or cup-like shape, in addition those where positive and negative curvatures alternate. An example of a sheet with Eigen Deformations allowed for the symmetry group of order 2 is shown in Fig. 4. Shear strains are invariant with rotations by $\pi$. Thus a thin sheet made of orthotropically aligned fibers may have Eigen Deformations in shear, regardless whether the fiber alignment orthotropy is two- or three-dimensional.

\subsection{Thin Sheets with Square-Symmetric Orientation of Fibers}


For a structure with all fibers parallel to a plane and with square-symmetric orientation distribution of fibers within the plane, in addition to three-dimensional orthotropy in fiber alignment, the symmetry group is $8 / 4$. The group contains three in-plane rotations, and four out-of-plane rotations. Any out-of plane rotation converts any concave curvature convex, and vice versa. The angular separation between the axis of the four out-of-plane rotations is $\pi / 4$. Within any such axis, the curvature changes sign, and shows sign-reversal symmetry with respect to the axis. On the other hand, the in-plane rotations require the same kind of curvature to appear at angular intervals of $\pi / 2$. The initially flat structure does not need to remain flat in Eigen Deformations, but it may have periodic out-of-plane deformations with an angular period of $\pi / 2$. Such deformations must be symmetric, except for sign reversal, with respect to in-plane rotation with an angle which is the angular separation between an original position and any line of out-of-plane rotation symmetry multiplied by -2 . An example of a sheet with Eigen Deformations allowed for group $8 / 4$ is shown in Fig. 5 . Shape shown in Fig. 3 would not be symmetric enough for this group - Fig. 5 illustrates the minimum of symmetries for Eigen Deformations within 8/4.

Within a structure with all fibers parallel to a plane and with square-symmetric orientation distribution of fibers within the plane, the fiber alignment is not necessarily orthotropic in three dimensions. In such a case out out-of plane rotations are not symmetry operations for the sheet, and the symmetry group is $4 / 1$. In such a case Eigen Deformations may produce a body which has the shape of a cup, or a paraboloid, or any structure where similar pattern of deformation is found at angular distances of $\pi / 2$. An example of a sheet with Eigen Deformations allowed for group 4/1 is shown in Fig. 6. Shape shown in Fig. 4 would not be symmetric enough for this group- Fig. 6 illustrates the minimum of out-of-plane symmetries for Eigen Deformations within 4/1.

Any shear strain within a plane perpendicular to the rotation axis changes its sign with a rotation by $\pi / 2$. Thus such a rotation, as a material symmetry operation, does not allow shear strain within a small volume element with essentially homogeneous strain state. However, in the case of a macroscopic body with non-homogenous strain state, shear strains may appear. The just need to change their sign at angular intervals of $\pi / 2$. An example of such a two-dimensionally deformed structure is shown in Fig. 7. Fig. 8 shows the combination of in-plane and out-ofplane deformations within group $8 / 4$.

\subsection{Thin Sheets with Isotropic In-Plane Orientation of Fibers}

For a structure with all fibers parallel to a plane and with isotropic orientation distribution of fibers within the plane, the symmetry was above described in terms of discrete symmetry group $12 / 3$, which corresponds to in-plane quasi-isotropy in terms of a 60/0/60 - laminate. However, there are more symmetry operations: any rotation within the plane is a symmetry operation for this kind of a structure. An effect always being at least as symmetric as the cause, Eigen Deformations always retain the symmetries of the structure. Thus a thin sheet with isotropic fiber orientation distribution within the plane always retains in-plane rotation symmetries. One consequence is that such a body cannot have in-plane Eigen Deformations in shear.

In-plane rotation symmetries being retained does not necessarily mean that the sheet remains planar. Even a conical or paraboloidal body has such rotation symmetries. However, the symmetry group $12 / 3$ contains also out-of-plane rotations as symmetry operations. These being retained in Eigen Deformations does assure that the sheet remains planar. 
W ithin a structure with all fibers parallel to a plane and with isotropic orientation distribution of fibers within the plane, the fiber alignment is not necessarily orthotropic in three dimensions. In other words, the in-plane isotropy is not necessarily invariant as a function of the co-ordinate perpendicular to the plane, since axial fiber rotation may differ. Then, out-of plane body rotations are not symmetry operations for the sheet, and the symmetry group corresponding to the $60 / 0 / 60$ - laminate is $6 / 1$. In such a case Eigen Deformations may produce a body which has the shape of a cup or a paraboloid. Deformed sheets shown in Figs. 4 and 6 are not symmetric enough to describe two-dimensional fiber alignment isotropy, which is illustrated in Fig. 9.

\section{Discussion}

The results indicate that plane reflection and inversion symmetries being absent, the order of any symmetry group becomes reduced by a half. Any rotation or line reflection can be produced in terms of two consequent plane reflections $[11,12$, 13]. Groups generated by reflections thus include rotations. On the other hand, groups generated by rotations do not include reflections.

The results also indicate that the degeneracies in stiffness Eigenvalues might always be the same for a body consisting of fibers with microfibrils as they are for a non-fibrous body with material symmetries corresponding to those of fiber alignment symmetries. Let us try to clarify the mechanisms behind these observations. They may be explainable in terms of vectors generating "point groups" as spatial symmetry groups.

In three-dimensional Euclidean space, any point group of spatial symmetry can be generated by three unit vectors. Let us denote the generating vectors $\mathbf{a}, \mathbf{b}$ and $\mathbf{c}$. In order to generate a dicyclic symmetry group, the three vectors must satisfy dicycle conditions [12] $(a b)^{p}=(b c)^{q}=(c a)^{r}=-1$, where the exponents refer to integers, and the product of any two vectors refers to the geometric product [11]. Explicit solutions for the geometric products are

$a b=e^{i c^{\prime} \pi / p}$
$b c=e^{i a^{\prime} \pi / q}$
$c a=e^{i b^{\prime} \pi / r}$.

In the case of three-dimensional symmetry, the spherical triangle with the generating unit vectors as vertices must have a positive area. The area is given by the spherical excess formula [11]

$$
\Delta=\pi\left(\frac{1}{p}+\frac{1}{q}+\frac{1}{r}-1\right)
$$

Then, the values of the integer parameters are restricted by

$$
\frac{1}{p}+\frac{1}{q}+\frac{1}{r} \succ 1
$$

We find that the smallest of the three integer parameters must be at most 2 , and the three generating vectors are independent only if all of the parameters are greater than unity. Thus the smallest one must equal 2, and Eq. (2) restricts the values of the rest. Let us denote the parameter " $r$ " to be 2 . Then the parameter space is characterized by the values of $p$ and $q$ [12]. If $q$ is 2 , any value of $p$ is allowed. If $q$ is 3 , possible values of $p$ are 3,4 and 5 .

Thus possible point groups in three-dimensional Euclidean space can be identified by notations $p$ (for two-dimensional symmetry) and $p q$. In particular, these notations refer to reflection groups, which include rotations. On the other hand, rotation 
groups do not include plane reflections. Groups missing some reflection symmetries are denoted as $\bar{p}, \overline{p q}, p \bar{q}, \bar{p} \bar{q}$, and $\overline{p q}$ [12].

Let us now discuss the reflection and rotation groups corresponding to orthotropic and fibrous materials. Transverse square-symmetry, in addition to orthotropy, corresponds to symmetry group $16 / 6$. It can be written $p q=42$. On the other hand, a body with all-parallel, transversely square-symmetric fibers corresponds to symmetry group $8 / 4$, which can be written $\overline{p q}=\overline{4} \overline{2}$. The latter symmetry group also applies to a fibrous structure with all fibers parallel to a plane, and with square symmetric orientation distribution within the plane, or to any three-dimensional fibrous body with orthotropic and transversely square-symmetric fiber alignment distribution. All these materials have two two-fold degeneracies in stiffness Eigenvalues, and thus five of the Eigenvalues are distinct.

Transverse quasi-isotropy, in addition to orthotropy, can be described in terms of symmetry group $24 / 4$. It can be written $p q=32$. On the other hand, a fibrous structure with all fibers parallel to a plane, and with quasi-isotropic orientation distribution within the plane corresponds to symmetry group 12/3, which can be written $\bar{p} \bar{q}=\overline{3} 2$. The same symmetry group applies to any fibrous body with orthotropic and transversely quasi-symmetric fiber alignment distribution. All these materials have three two-fold degeneracies in stiffness Eigenvalues, and thus four of the Eigenvalues are distinct.

Cube-symmetry corresponds to the full Octahedral group of order 48 , or $p q=43$. The symmetry group of a structure with cube-symmetric alignment of fibers is the rotational Octahedral group of order 24 , or $\overline{p q}=\overline{4} \overline{3}$. Both of these groups represented in nine-dimensional strain space show one two-fold and two three-fold degeneracies in stiffness Eigenvalues. Thus there are three distinct stiffness Eigenvalues.

The results indicate that the degeneracies in stiffness Eigenvalues might always be the same for a body consisting of fibers with microfibrils as they are for a nonfibrous body with material symmetries corresponding to those of fiber alignment symmetries. Stiffness degeneracies appear to be controlled by the integer exponents of dicycle conditions applied on products of vectors generating symmetry groups. In other words, it appears that stiffness degeneracies of a material with symmetry group $\overline{p q}$ are the same as those of a material with symmetry group $p q$.

It appears that fibrous materials show very different Eigen Deformations, in comparison to corresponding orthotropic materials. Plane reflection symmetries being absent, shear deformations may appear as Eigen Deformations, and thin sheets do not retain their planar shape. Regarding thin sheets, some experimental observations have been published recently $[19,20]$. A few other examples are familiar to anyone who has dried small wood specimens.

An effect may display more symmetries than the cause [7, 8]. The Eigen Deformations of a fibrous body significantly differing from those of a corresponding orthotropic body - being less symmetric - does require that at least one of the dimensions of the body is not very much greater than the thickness of a fiber. In the case of a large body, the spatial separation of two adjacent fiber walls becomes negligible in relation to the size of the entire structure, and thus the behavior of a large body approaches that of the corresponding orthotropic structure. 
Interestingly, the spatial symmetries of a structure are always retained in Eigen Deformations. The symmetries do not need to be related to fibrous structure: all features of the material, as well as the geometry of the body, affect the symmetries. For example, considering sawing and then drying of boards from tree trunks, a significant source of symmetry limitations is the alignment of growth rings. Boards with mutually perpendicular plane reflection symmetries will experience normal Eigen Deformations only, whereas boards with rotation symmetries only show shear deformations.

\section{References}

1. E. Noether, Nachr. König. Gesellsch. W iss. Göttingen, Math.-P hys. KI., 235-257 (1918)

2. M. Hamermesh, Group theory and its applications to physical problems (Addison-Wesley, Reading, MA, 1962).

3. M. Tinkham, Group theory and quantum mechanics (McGraw-Hill, NY, 1964)

4. P. Szekeres, A course in modern mathematical physics, pp. 27-58 (Cambridge, 2004)

5. K.F. Riley, M.P. Hobson, S.J. Bence, Mathematical methods for physics and engineering, 2. Edn, pp. 883-960. (Cambridge, 2002)

6. G.B. Arfken, H.J. Weber, Mathematical methods for physicists, 5. edn, pp. 273301. (Hartcourt, CT, 2001)

7. P. Curie, J ournal de Physique 3, 393-415 (1894). English translation: J. Rosen, P. Copie, in: "Symmetry in Physics: Selected Reprints", ed. J. Rosen, American Association of Physics Teachers, College Park, MD, 1982, pp. 17-25.

8. J. Rosen, Symmetry rules. How Science and Nature are founded on Symmetry. (Springer, 2008)

9. A.D. Thomas, G.W. Wood, Group Tables. (Shiva Publishing, Kent, UK, 1980).

10. G. Burns, A.M. Glazer, Space groups for solid state scientists. (Academic Press, NY, 1978)

11. D. Hestenes, New foundations for classical mechanics, 2. edn. (Kluwer, 1999)

12. D. Hestenes, in Applications of Geometric Algebra in Computer Science and Engineering, edited by L. Dorst, C. Doran, and J. Lasenby (Birkhauser, Boston, 2002), pp. 3-34.

13. D. Hestenes, J. Holt, J . Mathematical Physics. 48, 023514 (2007).

14. P.P. Kärenlampi, Submitted for publication, March 19, 2008.

15. J.D. Boyd, Wood Sci. Tech. 11, 3-22 (1977).

16. R.D. Preston, Royal Soc. Phil. Trans. B 224, 131-174, plates 17-18 (1934).

17. I.W. Bailey, M.R. Vestal, J. Arnold Arboretum 18(3), 185-195, Pls. 209-208

(1937).

18. R.A. Cockrell, Wood Sci Tech. 8, 197-206 (1974).

19. P.H. Viitaharju, K. Niskanen, J . Pulp Paper Sci. 20, 148-152 (1994).

20. R.C. Neagu, R.K. Gamstedt, M. Lindström, M., Composites A 36, 772-788 (2005). 
Petri P. K ärenlampi, A pril 27, 2009

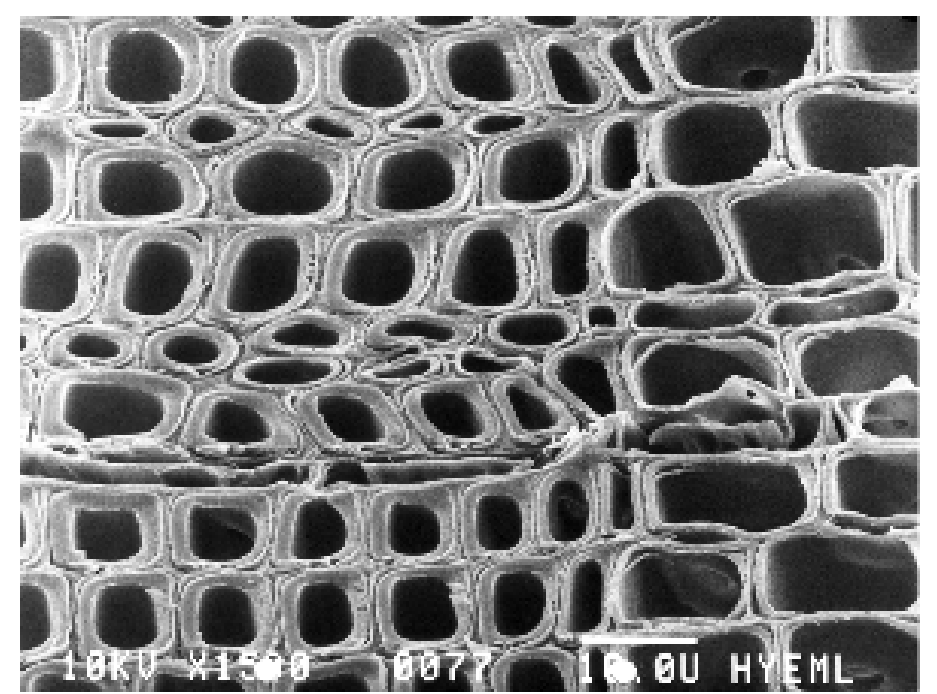

Figure 1. Cellular tissue of softwood. Courtesy of Pekka Saranpää.

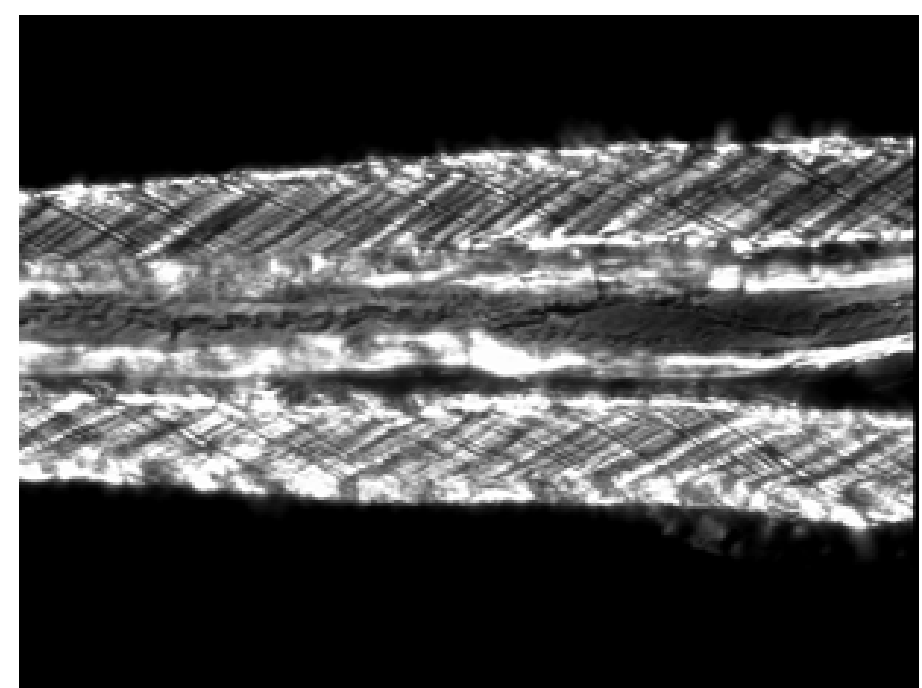

Figure 2. An individual fiber under polarized light. Courtesy of Pekka Saranpää. 


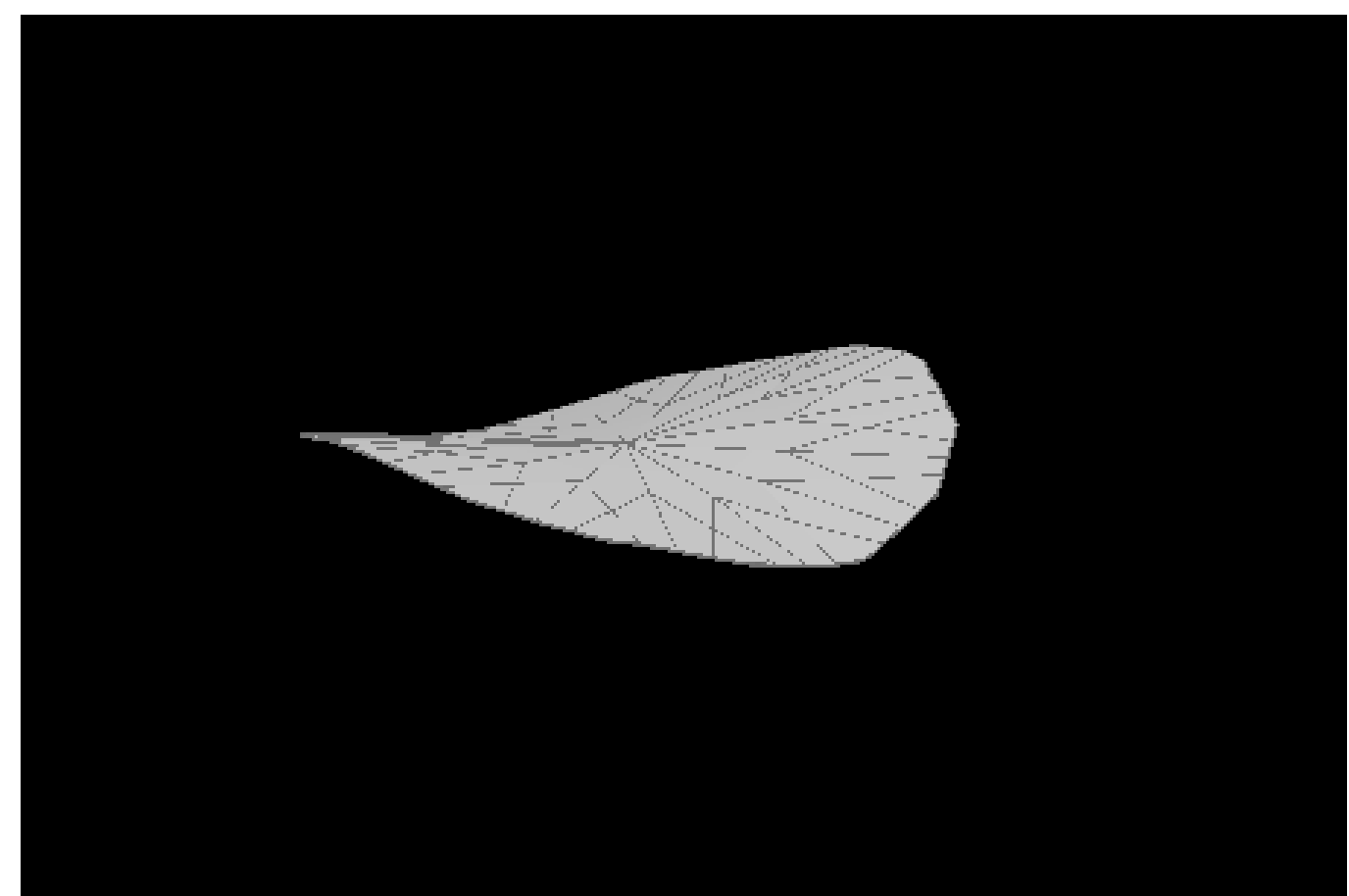

Figure 3. A saddle-shaped structure with out-of-plane deformations with angular period of $\pi$, with out-of plane rotation symmetries by $\pi$.

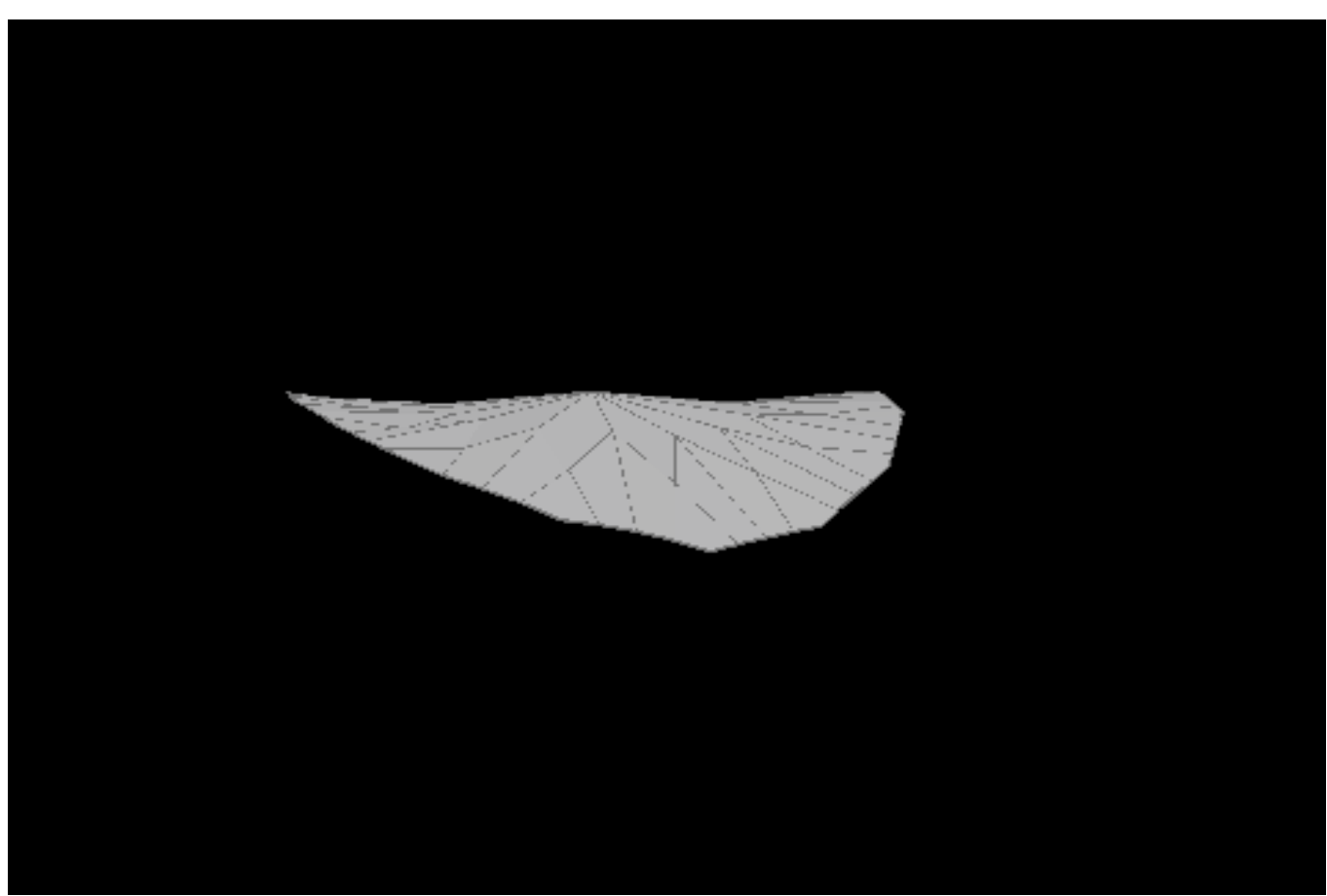

Figure 4. A structure with out-of-plane deformations with angular period of $\pi$, without out-of plane rotation symmetries. 


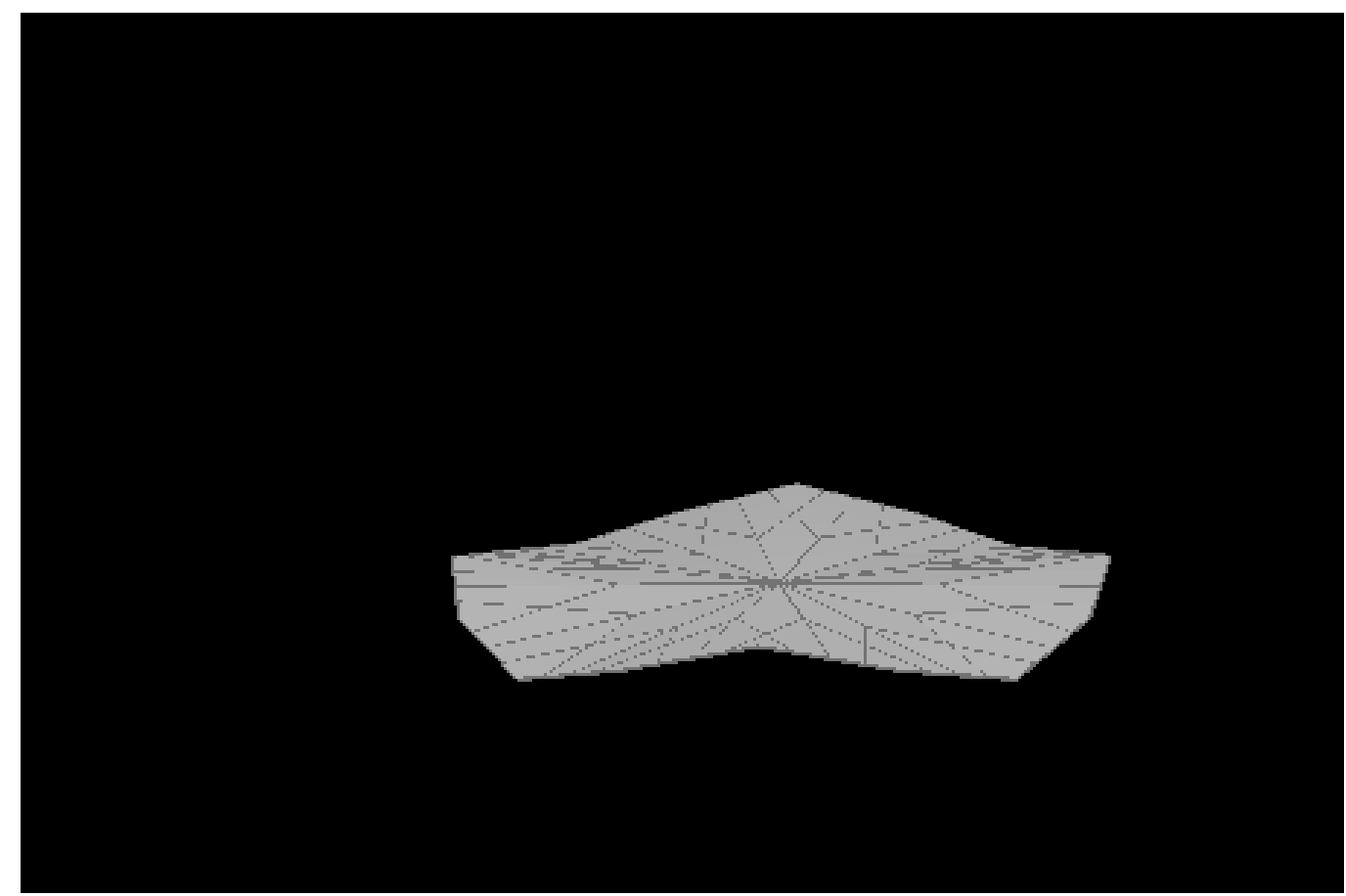

Figure 5. A structure with out-of-plane deformations with angular period of $\pi / 2$, with out-of plane rotation symmetries by $\pi$.

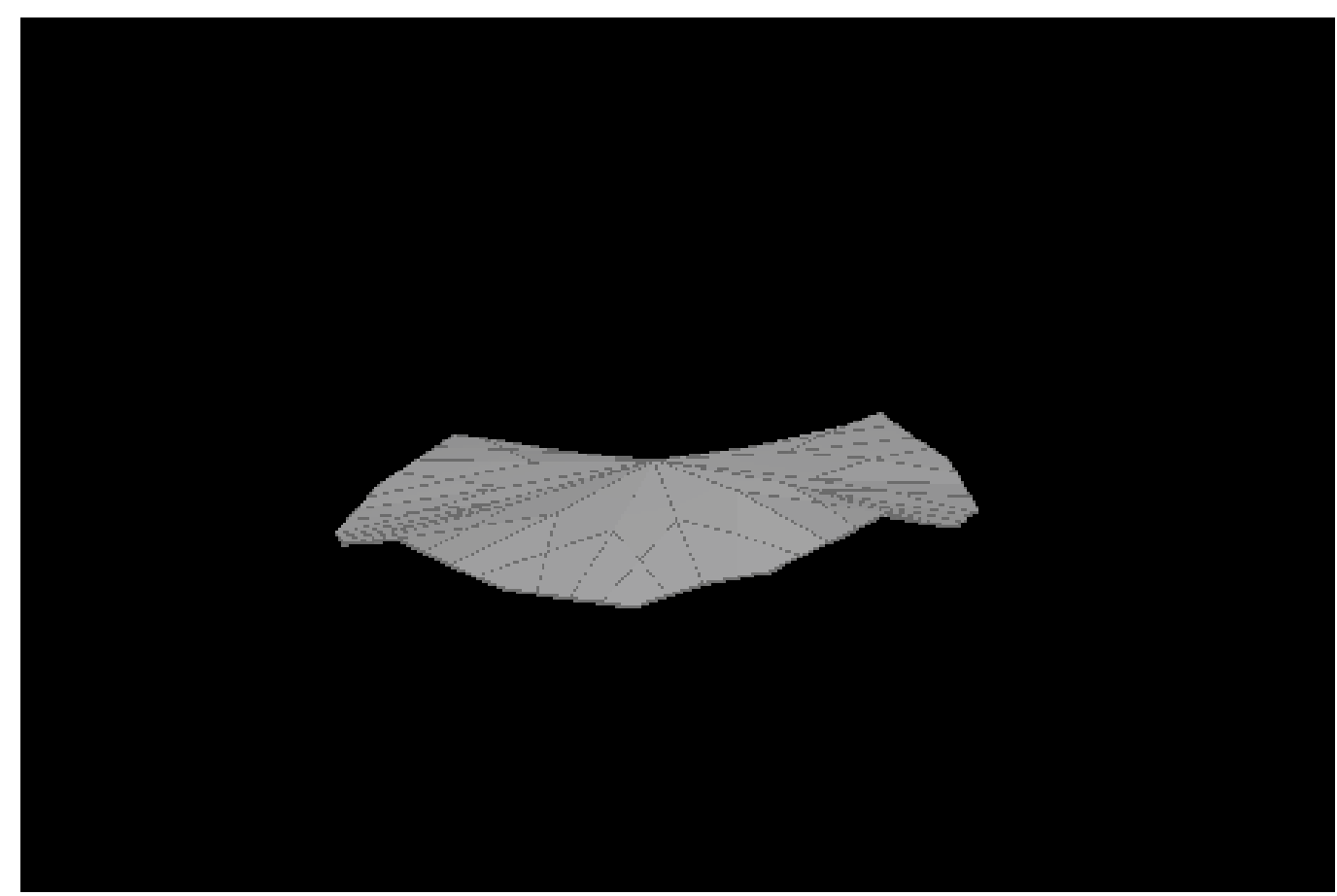

Figure 6. A structure with out-of-plane deformations with angular period of $\pi / 2$, without out-of plane rotation symmetries. 


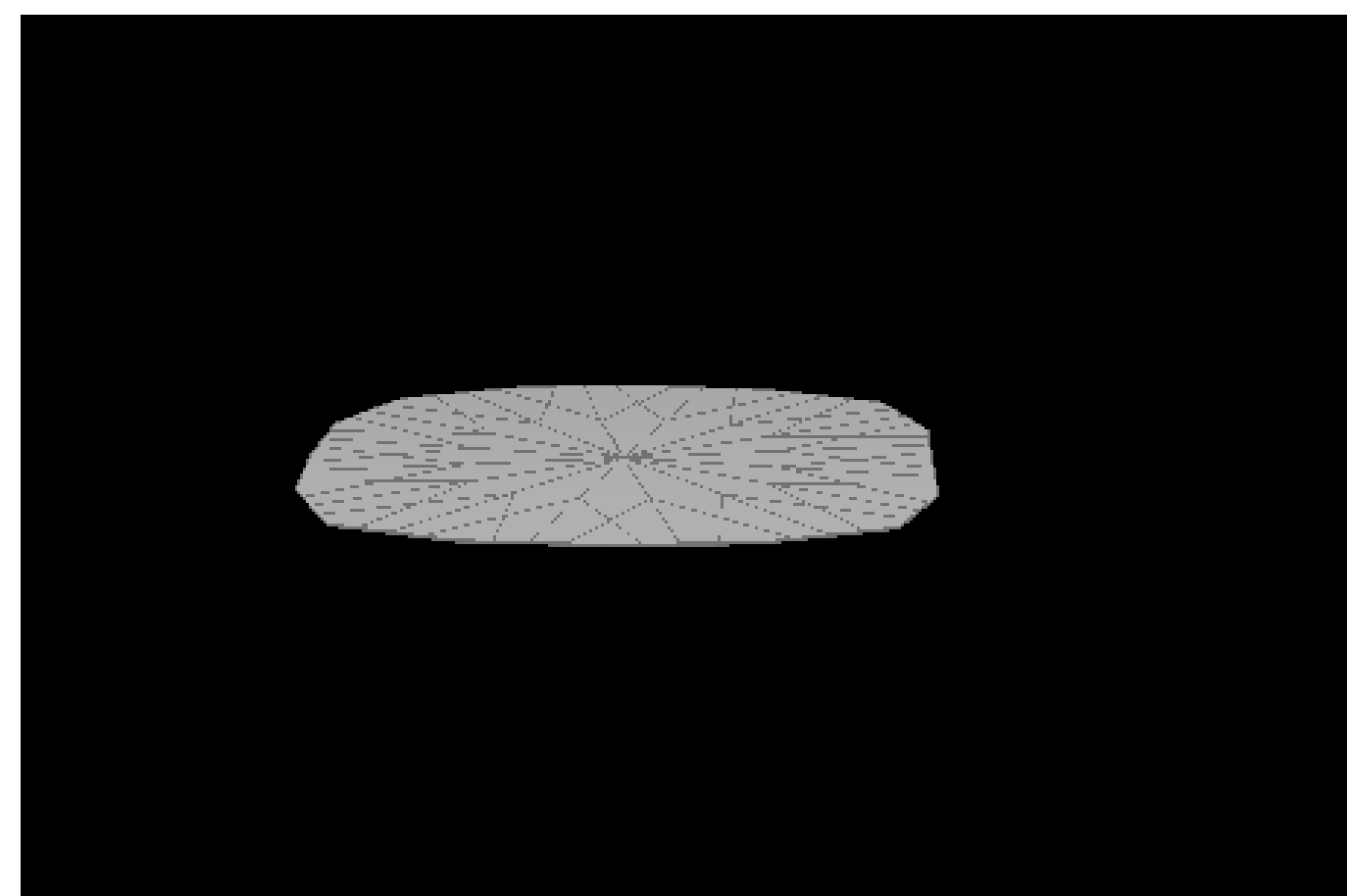

Figure 7. An initially round sheet, showing in-plane shear strain which changes sign along with rotation by $\pi / 2$.

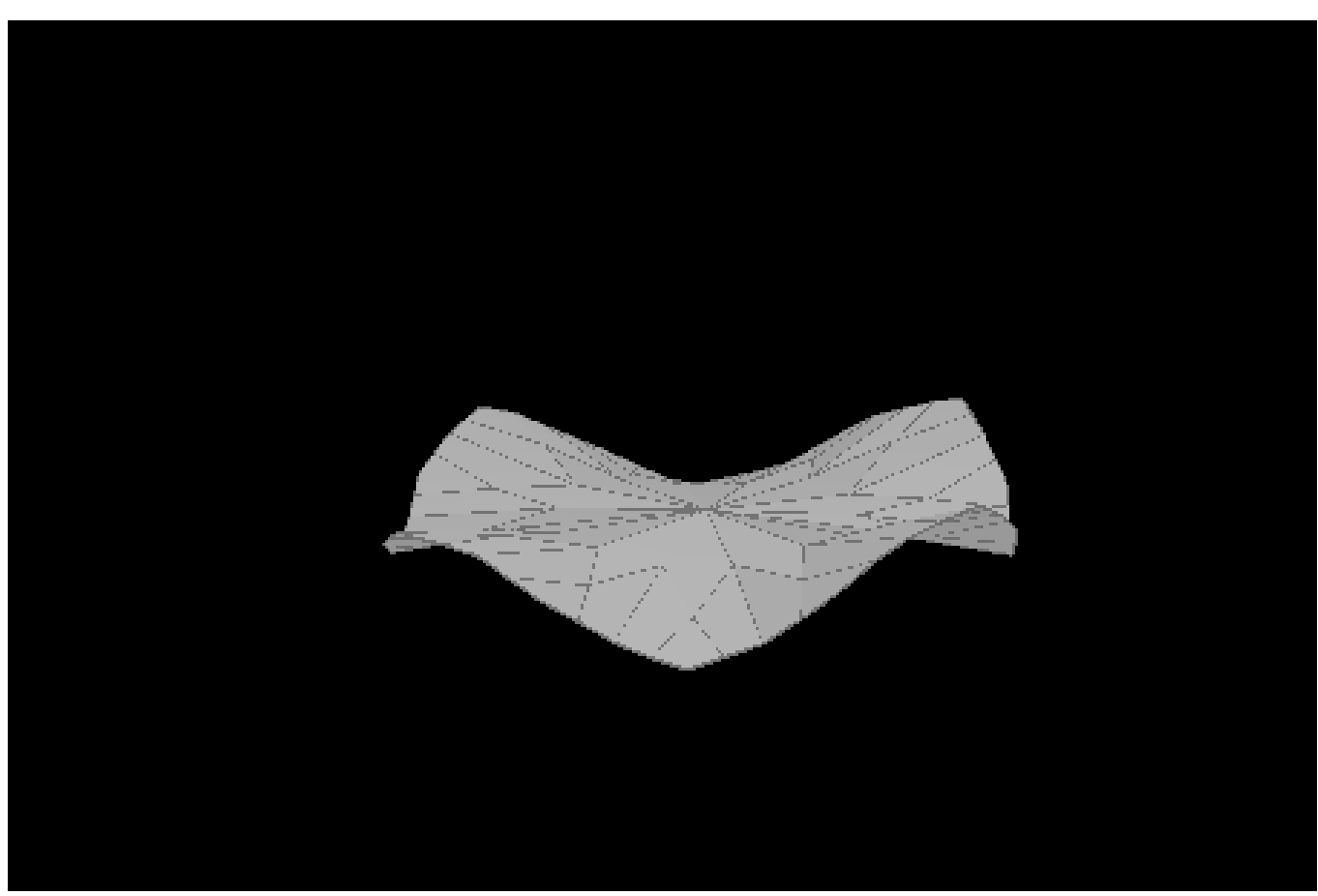

Figure 8. A combination of the deformations shown in Figs 5 and 7. 


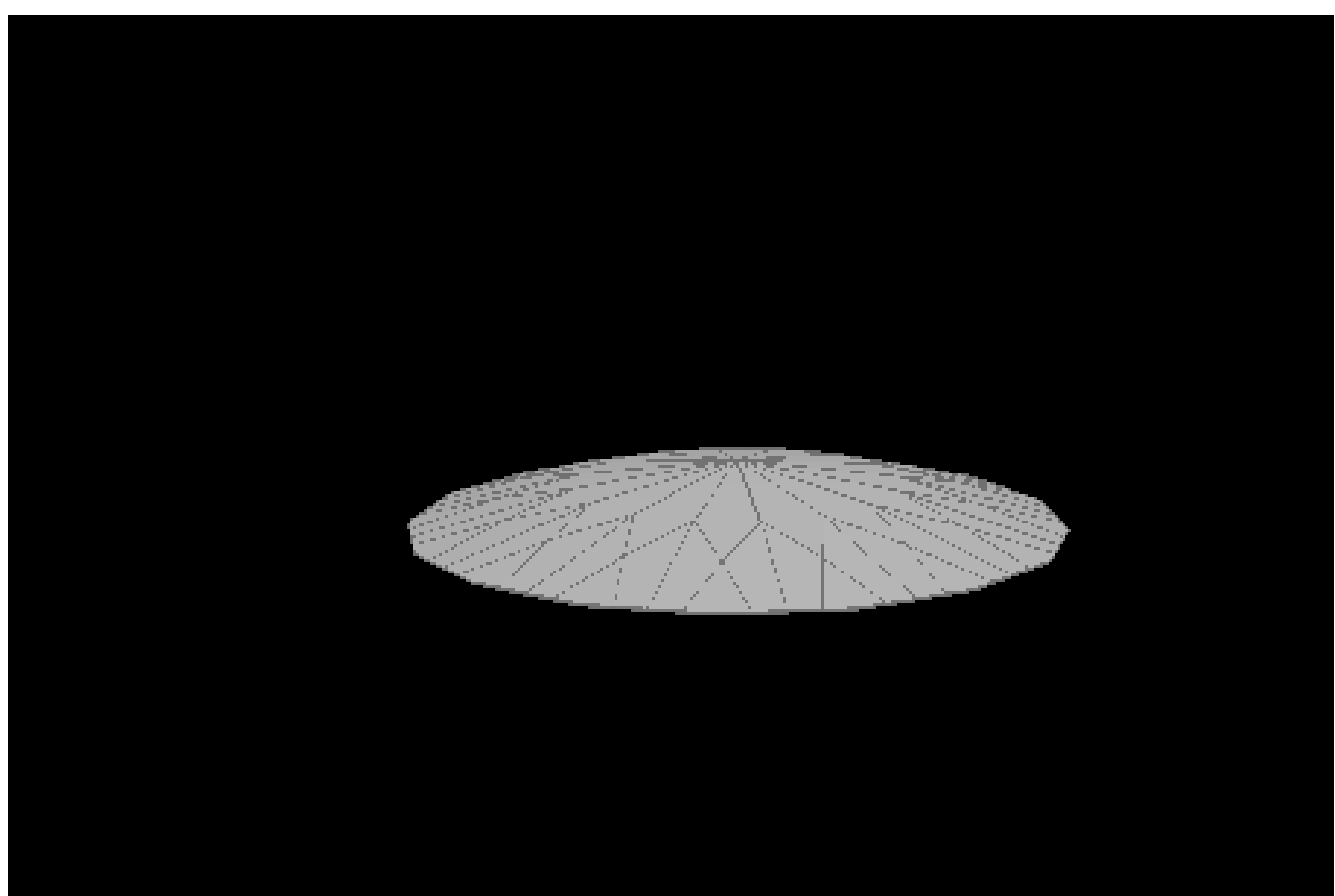

Figure 9. A cup-shaped structure, as a consequence or two-dimensional fiber alignment isotropy.

Table 1. Character Table for Group 16/6.

\begin{tabular}{|c|c|c|c|c|c|c|c|c|c|c|}
\hline & I & $2 \times 3$ & $\begin{array}{l}2 \times 1 \\
2 \times 2\end{array}$ & $\begin{array}{c}4_{\times 3} \\
4^{\prime} \times 3\end{array}$ & $M_{x 12}$ & $\begin{array}{l}M_{x 13} \\
M_{x 23}\end{array}$ & $\begin{array}{l}m_{x 13} \\
m_{x 23}\end{array}$ & $\mathrm{i}$ & $\begin{array}{l}\overline{4_{x 3}} \\
\overline{4_{x 3}^{\prime}}\end{array}$ & $\frac{m_{x 13}}{\overline{m_{x 23}}}$ \\
\hline$A_{1 a}$ & 1 & 1 & 1 & 1 & 1 & 1 & 1 & 1 & 1 & 1 \\
\hline$A_{2 q}$ & 1 & 1 & -1 & 1 & 1 & -1 & -1 & 1 & 1 & -1 \\
\hline$B_{1 q}$ & 1 & 1 & 1 & -1 & 1 & 1 & -1 & 1 & -1 & -1 \\
\hline$B_{2 g}$ & 1 & 1 & -1 & -1 & 1 & -1 & 1 & 1 & -1 & 1 \\
\hline $\mathrm{E}_{\mathrm{q}}$ & 2 & -2 & 0 & 0 & -2 & 0 & 0 & 2 & 0 & 0 \\
\hline$A_{1 u}$ & 1 & 1 & 1 & 1 & -1 & -1 & -1 & -1 & -1 & 1 \\
\hline$A_{2 u}$ & 1 & 1 & -1 & 1 & -1 & 1 & 1 & -1 & -1 & -1 \\
\hline$B_{1 u}$ & 1 & 1 & 1 & -1 & -1 & -1 & 1 & -1 & 1 & -1 \\
\hline$B_{2 u}$ & 1 & 1 & -1 & -1 & -1 & 1 & -1 & -1 & 1 & 1 \\
\hline $\mathrm{E}_{\mu}$ & 2 & 2 & 0 & 0 & 2 & 0 & 0 & -2 & 0 & 0 \\
\hline
\end{tabular}


Table 2. Character Table for Group $O_{h}$.

\begin{tabular}{|c|c|c|c|c|c|c|c|c|c|c|}
\hline & I & $\begin{array}{l}2 \times 1 \\
2 \times 2 \\
2 \times 3\end{array}$ & $\begin{array}{l}4_{x 1} \\
4^{\prime}{ }_{x 1} \\
4_{x 2} \\
4^{\prime}{ }^{\prime}{ }_{x 2} \\
4_{x 3} \\
4^{\prime}{ }_{x 3}^{\prime}\end{array}$ & ${ }^{6 *} C_{2}$ & $8^{*} C_{3}$ & $\begin{array}{l}M_{x 12} \\
M_{x 13} \\
M_{x 23}\end{array}$ & $\begin{array}{l}m_{x 13} \\
m_{x 23} \\
m_{x 12} \\
m_{x 32} \\
m_{x 21} \\
m_{x 31}\end{array}$ & $i$ & $\begin{array}{r}\text { i } 4_{x 1} \\
\text { i } 4^{\prime} \\
\text { i } 4_{x 2} \\
\text { i } 4_{x 2}^{\prime} \\
\text { i } 4_{x 3} \\
\text { i } 4^{\prime}\end{array}$ & $\begin{array}{c}8^{*} \\
\text { i C }_{3}\end{array}$ \\
\hline$A_{1 q}$ & 1 & 1 & 1 & 1 & 1 & 1 & 1 & 1 & 1 & 1 \\
\hline$A_{2 g}$ & 1 & 1 & -1 & -1 & 1 & 1 & -1 & 1 & -1 & 1 \\
\hline$E_{g}$ & 2 & 2 & 0 & 0 & -1 & 2 & 0 & 2 & 0 & -1 \\
\hline$T_{1 q}$ & 3 & -1 & 1 & 1 & 0 & -1 & -1 & 3 & 1 & 0 \\
\hline $\mathrm{T}_{2 \mathrm{q}}$ & 3 & -1 & -1 & -1 & 0 & -1 & 1 & 3 & -1 & 0 \\
\hline$A_{1 u}$ & 1 & 1 & 1 & 1 & 1 & -1 & -1 & -1 & -1 & -1 \\
\hline$A_{2 u}$ & 1 & 1 & -1 & -1 & 1 & -1 & 1 & -1 & 1 & -1 \\
\hline$E_{u}$ & 2 & 2 & 0 & 0 & -1 & -2 & 0 & -2 & 0 & 1 \\
\hline$T_{1 u}$ & 3 & -1 & 1 & 1 & 0 & 1 & 1 & -3 & -1 & 0 \\
\hline$T_{2 u}$ & 3 & -1 & -1 & -1 & 0 & 1 & -1 & -3 & 1 & 0 \\
\hline
\end{tabular}

Table 3. Character Table for Group 12/3.

\begin{tabular}{|c|c|c|c|c|c|c|}
\hline & I & $2 \times 3$ & $3_{x 3} \quad 3_{x 3}^{\prime}$ & $6_{x 3} 6_{x 3}^{\prime}$ & $M_{1} M_{2} M_{3}$ & $m_{1} m_{2} m_{3}$ \\
\hline$A_{1}$ & 1 & 1 & 1 & 1 & 1 & 1 \\
\hline$A_{2}$ & 1 & 1 & 1 & 1 & -1 & -1 \\
\hline$B_{1}$ & 1 & -1 & 1 & -1 & 1 & -1 \\
\hline$B_{2}$ & 1 & -1 & 1 & -1 & -1 & 1 \\
\hline$E_{1}$ & 2 & -2 & -1 & 1 & 0 & 0 \\
\hline$E_{2}$ & 2 & 2 & -1 & -1 & 0 & 0 \\
\hline
\end{tabular}

Table 4. Character Table for Group 24/4.

\begin{tabular}{|c|c|c|c|c|c|c|c|c|c|c|c|c|}
\hline & I & $2 \times 3$ & $\begin{array}{c}3 \times 3 \\
3^{\prime} \times 3\end{array}$ & $\begin{array}{c}6_{x 3} \\
6^{6}{ }^{\prime} 33\end{array}$ & $\begin{array}{l}M_{1} \\
M_{2} \\
M_{3}\end{array}$ & $\begin{array}{l}\mathrm{m}_{1} \\
\mathrm{~m}_{2} \\
\mathrm{~m}_{3}\end{array}$ & $M_{x 12}$ & $\mathrm{i}$ & $\begin{array}{c}\overline{\overline{3_{x 3}}} \\
\overline{\overline{3_{x 3}^{\prime}}}\end{array}$ & $\begin{array}{c}\overline{\overline{\sigma_{x 3}}} \\
\overline{\overline{6_{x 3}^{\prime}}}\end{array}$ & $\frac{\frac{2 \times 2}{\overline{M_{2}}}}{\overline{\overline{M_{3}}}}$ & $\begin{array}{l}m_{1} \\
2_{x 1} \\
m_{3}\end{array}$ \\
\hline$A_{1 a}$ & 1 & 1 & 1 & 1 & 1 & 1 & 1 & 1 & 1 & 1 & 1 & 1 \\
\hline $\mathrm{A}_{2 q}$ & 1 & 1 & 1 & 1 & -1 & -1 & 1 & 1 & 1 & 1 & -1 & -1 \\
\hline$B_{1 q}$ & 1 & -1 & 1 & -1 & -1 & 1 & -1 & 1 & -1 & 1 & -1 & 1 \\
\hline$B_{2 q}$ & 1 & -1 & 1 & -1 & 1 & -1 & -1 & 1 & -1 & 1 & 1 & -1 \\
\hline$E_{1 q}$ & 2 & -2 & -1 & 1 & 0 & 0 & -2 & 2 & 1 & -1 & 0 & 0 \\
\hline$E_{2 q}$ & 2 & 2 & -1 & -1 & 0 & 0 & 2 & 2 & -1 & -1 & 0 & 0 \\
\hline$A_{1 u}$ & 1 & 1 & 1 & 1 & -1 & -1 & -1 & -1 & -1 & -1 & 1 & 1 \\
\hline$A_{2 u}$ & 1 & 1 & 1 & 1 & 1 & 1 & -1 & -1 & -1 & -1 & -1 & -1 \\
\hline$B_{1 u}$ & 1 & -1 & 1 & -1 & 1 & -1 & 1 & -1 & 1 & -1 & -1 & 1 \\
\hline$B_{2 u}$ & 1 & -1 & 1 & -1 & -1 & 1 & 1 & -1 & 1 & -1 & 1 & -1 \\
\hline$E_{2 u}$ & 2 & -2 & -1 & 1 & 0 & 0 & 2 & -2 & -1 & 1 & 0 & 0 \\
\hline$E_{2 u}$ & 2 & 2 & -1 & -1 & 0 & 0 & -2 & -2 & 1 & 1 & 0 & 0 \\
\hline
\end{tabular}


Table 5. Character Table for Group 8/4.

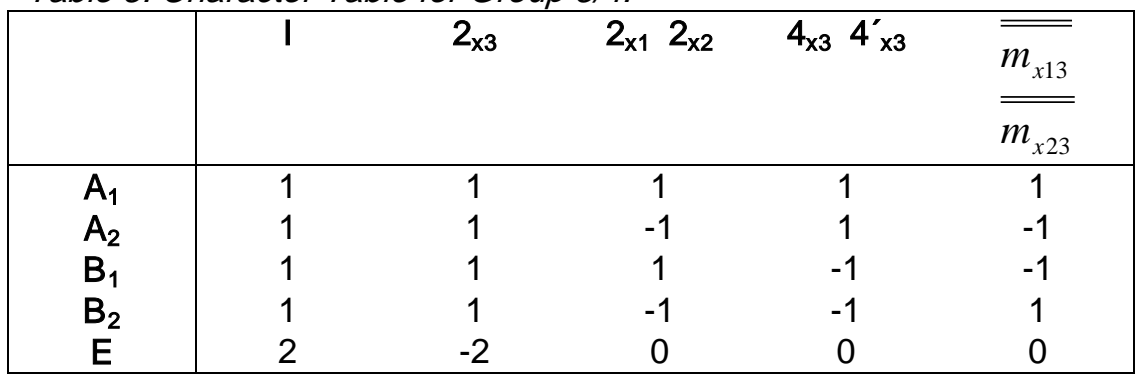

Table 6. Character Table for Group 12/3.

\begin{tabular}{|c|c|c|c|c|c|c|}
\hline & 1 & $2 \times 3$ & $3 \times 33_{x 3}^{\prime}$ & $6_{x 3} 6_{x 3}^{\prime}$ & $2_{x 1} L_{12} L_{12}^{\prime}$ & $2 \times 2 L_{6} L_{6}^{\prime}$ \\
\hline$A_{1}$ & 1 & 1 & 1 & 1 & 1 & 1 \\
\hline $\mathrm{A}_{2}$ & 1 & 1 & 1 & 1 & -1 & -1 \\
\hline $\mathrm{B}_{1}$ & 1 & -1 & 1 & -1 & 1 & -1 \\
\hline$B_{2}$ & 1 & -1 & 1 & -1 & -1 & 1 \\
\hline$E_{1}$ & 2 & -2 & -1 & 1 & 0 & 0 \\
\hline$E_{2}$ & 2 & 2 & -1 & -1 & 0 & 0 \\
\hline
\end{tabular}

